\title{
EDITORIAL
}

\section{Pediatric neurosurgery along with children's hospitals' innovations are rapid and uniform in response to the COVID-19 pandemic}

\author{
${ }^{*}$ Howard L. Weiner, MD, ${ }^{1}$ P. David Adelson, MD, ${ }^{2}$ Douglas L. Brockmeyer, MD, ${ }^{3}$ \\ Cormac O. Maher, MD, ${ }^{4}$ Nalin Gupta, MD, PhD, ${ }^{5}$ Matthew D. Smyth, MD, ${ }^{6}$ Andrew Jea, MD, \\ Jeffrey P. Blount, MD, ${ }^{8}$ Jay Riva-Cambrin, MD, MSc, ${ }^{9}$ Sandi K. Lam, MD, MBA, ${ }^{10}$ \\ Edward S. Ahn, MD, ${ }^{11}$ Gregory W. Albert, MD, MPH, ${ }^{12}$ and Jeffrey R. Leonard, MD ${ }^{13}$
}

\begin{abstract}
1Department of Neurosurgery, Division of Pediatric Neurosurgery, Texas Children's Hospital, Baylor College of Medicine, Houston, Texas; ${ }^{2}$ Department of Neurosurgery, Barrow Neurological Institute at Phoenix Children's Hospital, University of Arizona College of Medicine, Phoenix, Arizona; ${ }^{3}$ Department of Neurosurgery, Division of Pediatric Neurosurgery, Primary Children's Hospital, University of Utah, Salt Lake City, Utah; ' ${ }^{4}$ Department of Neurosurgery, Division of Pediatric Neurosurgery, Mott Children's Hospital, University of Michigan, Ann Arbor, Michigan; ' 5 Department of Neurological Surgery, Division of Pediatric Neurosurgery, UCSF Benioff Children's Hospital, University of California, San Francisco, California; ${ }^{6}$ Department of Neurological Surgery, Division of Pediatric Neurological Surgery, St. Louis Children's Hospital, Washington University School of Medicine in St. Louis, Missouri; ' ${ }^{7}$ Department of Neurosurgery, Division of Pediatric Neurosurgery, Riley Hospital for Children at IU Health, Indiana University School of Medicine, Indianapolis, Indiana; ${ }^{8}$ Department of Neurosurgery, Division of Pediatric Neurosurgery, Children's of Alabama, The University of Alabama at Birmingham School of Medicine, Birmingham, Alabama; ${ }^{9}$ Department of Clinical Neurosciences, Section of Neurosurgery, University of Calgary, Alberta, Canada; ${ }^{10}$ Department of Neurological Surgery, Division of Pediatric Neurosurgery, Ann and Robert H. Lurie Children's Hospital of Chicago, Northwestern University Feinberg School of Medicine, Chicago, Illinois; ${ }^{11}$ Department of Neurosurgery, Division of Pediatric Neurosurgery, Children's Center, Mayo Clinic College of Medicine and Science, Rochester, Minnesota; ${ }^{2}$ Department of Neurosurgery, Division of Pediatric Neurosurgery, Arkansas Children's Hospital, University of Arkansas for Medical Sciences, Little Rock, Arkansas; and ${ }^{13}$ Department of Neurological Surgery, Section of Neurosurgery, Nationwide Children's Hospital, The Ohio State University College of Medicine, Columbus, Ohio
\end{abstract}

$\mathrm{T}$ HE COVID-19 pandemic is an unprecedented international crisis, with enormous health, economic, and social consequences, the likes of which have not been experienced in recent human history. The global medical community is facing historic practical and ethical challenges that require an urgent response. This situation has necessitated swift decision-making in the neurosurgery community. An inspiring and consistent message emerged from the resultant conversation, demonstrating our discipline's response to this tragedy, which was strikingly rapid, uniform, and thoughtful across the many centers represented. Not surprisingly, pediatric neurosurgeons are able to lead their teams through this unprecedented time with creative decision-making and a razorsharp focus on the health of both patients and colleagues. At the time of this writing, each center reported either no or only a small number of COVID-19-positive cases at their pediatric hospital, among either patients or staff, and a range of documented coronavirus community transmissions. Several notably consistent themes emerged from this exchange among the 13 members of the Journal of Neurosurgery: Pediatrics Editorial Board, the common concept being that decision-making remains dynamic and is modified as needed on a daily basis.

\section{Pediatric Neurosurgical Procedures}

Consistently at every center, nearly immediately, the decision to perform pediatric neurosurgical procedures followed the American College of Surgeons Clinical Issues and Guidance for the triage and management of elective surgical procedures (https://www.facs.org/covid-19/ clinical-guidance), and the procedures were limited to only those defined as urgent. The rationale behind this decision was to preserve supplies, personal protective equipment (PPE), blood products, and ICU beds and to minimize exposure of the surgery, anesthesia, and operating room personnel to potential COVID-19-positive patients. Although "urgent" was defined in some centers as a case that could not be delayed for 14 days, others were triaged 
as a case that could not be delayed for 30 or more days. In many centers, a three-tiered system has been utilized to classify procedures as follows: 1) essential/emergency cases - if not done immediately, death or disability would result (e.g., trauma and acute intracranial hemorrhage with mass effect, shunt malfunction, myelomeningocele repair, baclofen withdrawal, spinal cord compression, and acute hydrocephalus); 2) essential/urgent-time sensitive (e.g., craniotomy for newly diagnosed or progressive tumor); and 3) elective cases that can safely be rescheduled 30 or more days later in most cases (e.g., craniofacial surgery, Chiari decompression, baclofen pump placement, rhizotomy, and tethered cord release). To limit the use of supplies, only essential team members were allowed to operate, most often with the exclusion of "learners." Most hospitals have limited the number of operating rooms potentially in use; however, centers have differed on the requirements for PPE during the surgical procedure, with most requiring the use of fitted N95 masks for personnel present during intubation and extubation and, in some instances, personnel have been asked to leave the room during intubation. A range of policies have been in effect regarding the requirement for testing patients for COVID-19 prior to surgery. Some centers distribute limited supplies, such as one mask per day to each surgeon.

\section{Clinic}

One of the most immediate and significant consequences of the COVID-19 pandemic was the universal expansion of telemedicine as an emerging way to conduct patient clinic visits. Workflow optimization, training, billing, and credentialing for both telephone and video-based clinic visits occurred very quickly at all centers, limiting in-person visits to only those deemed as urgent and absolutely necessary, the rationale being to minimize traffic into the facility of patients and family members who may be COVID-19 positive and face-to-face interaction with staff and clinic personnel. Initially, clinic visits deemed as routine follow-ups were rescheduled to a later date; however, it became apparent that full clinic schedules could be maintained with telemedicine capability. In order to limit neuroradiology studies to only those that are deemed urgent and necessary, surgeons have been asked to work with their radiology colleagues, frequently reviewing upcoming imaging sessions, and to reschedule studies that do not meet criteria for "urgent."

\section{Faculty}

All centers described a reorganization of the faculty members into "teams" of at least two faculty members who would rotate, most often, on a weekly basis, with some rotating either for 3-4 days at a time or daily. For example, a common arrangement is for two surgeons to rotate call every other day for 1 week at a time, while the second team remains off-site at home, still participating in virtual team conferences, clinical research, or telemedicine clinic visits. The rationale behind this was to minimize contact between faculty surgeons as well as minimizing the number at risk at any one time and, thereby, reduce the risk of potential COVID-19 exposure. These arrangements, which call for faculty members to remain away from the hospital setting, have been particularly challenging, as they are contrary to the normal work ethic and commitment of our pediatric neurosurgery community members.

\section{Residents, Fellows, and Advanced Practice Providers}

Resident pediatric neurosurgery rotations have also been changed significantly, with the house staff divided into distinct teams, maintaining what resembles a "weekend" manpower schedule. The most-frequently cited arrangement has two pediatric neurosurgery residents alternating 24-hour call each, on an every-other-day rotating basis. The resident who is not on call remains at home. The rationale for this is to minimize resident exposure to potential COVID-19-positive patients. Advanced practice providers (APPs) are also arranged into rotating teams in order to keep personnel out of the hospital as much as possible. In addition, APPs at some centers with predominantly ambulatory practice began conducting telemedicine or telephonic visits as possible, providing an opportunity to keep staff away from the hospital setting. A variety of arrangements were cited for the pediatric neurosurgery fellow rotating in the schedule. A concern was expressed about the potential impact of this pandemic on fellow and resident training, particularly if fellows and residents were not able to fulfill case index requirements due to the changes in the operative volume and experience. This will likely not be an issue at large-volume programs but potentially impacts those fellowships with low operative volumes.

\section{Conferences}

Daily morning report, educational, and subspecialty conferences (for example, epilepsy, craniofacial, tumor board, and spine) and journal club for the entire pediatric neurosurgery team are conducted electronically by telephone or video conference. Most groups also conduct a daily telephone call or video conference, led by service chiefs, to keep their entire teams, including faculty, house staff, APPs, nurses, research, and administrative staff, updated on the rapidly evolving information on the COVID-19 pandemic. Most hospitals also conduct virtual administrative meetings in a similar fashion. Administrative and research staff are limited, with most working from home.

\section{Screening and PPE}

Several centers reported a daily screening procedure before individuals are allowed to enter the hospital facility each day, consisting of screening questions about travel history, symptoms, and potential virus exposure, and at which time body temperature is assessed. Some facilities have also required N95 mask fit testing for all surgeons.

\section{The Silver Lining}

This global pandemic, while causing significant strains on the national and local healthcare systems, economies, delivery of patient care, and, most importantly, on our patient interactions, has precipitated a rapid integration of new paradigms that will likely have a positive impact as 
they are developed for the future. The largest of these will be the implementation of more advanced telemedicine that will potentially benefit our healthcare systems, healthcare workers, and patients and their families. Similarly, changing the ways in which we do business, providing more opportunities for "off-campus" work to be done, has great implications for reducing the need for on-campus facilities for education and research, providing more flexible schedules for patient access and potentially improving the work-life balance for staff and pediatric neurosurgery personnel. The more widespread use of video conferencing has the potential for opening up further opportunities not only for greater local exchange and interaction but also particularly for neurosurgical/neuroscience education and collaborative research nationally and globally for scientific exchange and knowledge. Despite this "silver lining," the price of this pandemic remains too high. At the time of this writing, we learned of the tragic passing of our colleague Dr. James T. Goodrich, a major neurosurgery casualty of COVID-19.

https://thejns.org/doi/abs/10.3171/2020.4.PEDS20240

\section{Disclosures}

The authors report no conflict of interest.

\section{Correspondence}

Howard L. Weiner: hlweiner@texaschildrens.org.

INCLUDE WHEN CITING

Published online April 17, 2020; DOI: 10.3171/2020.4.PEDS20240. 